\title{
Study of the Comparison of Endoscopic Closure and Laparoscopic Repair of Defect of Gastric Wall
}

\section{Qiao Qiao}

Affiliated Hospital of Jiangnan University

Huiming Tu ( $\square$ tuhuimingcn@163.com )

Affiliated Hospital of Jiangnan University

\section{Bojian Fei}

Affiliated Hospital of Jiangnan University

Kebin Xu

Affiliated Hospital of Jiangnan University

Fan Yang

Affiliated Hospital of Jiangnan University

Jie Li

Affiliated Hospital of Jiangnan University

Qizhong Gao

Affiliated Hospital of Jiangnan University

\section{Research article}

Keywords: Gastric Mucosal Tumor, Intrinsic Muscle Layer Tumor, Endoscopic Submuscular Dissection, Endoscopic Resection, Therapeutic Perforation, Laparoscope Assisted Gastroscope

Posted Date: November 13th, 2020

DOI: https://doi.org/10.21203/rs.3.rs-104761/v1

License: (c) (i) This work is licensed under a Creative Commons Attribution 4.0 International License.

Read Full License 


\section{Abstract}

Objective: To make analysis on the curative effect on the technique of endoscopic closure and laparoscopic closure, and explore the effect and safety of acute perforation of the endoscopic closure technique.

Methods: Analysis on the 40 therapeutic cases of laparoscopic repair and endoscopic closure treatments, respectively, to submucosal tumours (SMT) of the stomach removed through ESE/EFR. According to the double-sample t-test, the differences of operation time and medical expenses between the two closure methods were compared and analyzed. And used the chi-square test to compare the difference in terms of operational difficulty and effects.

Results: Postoperative pathology of 76 therapeutic cases operated through ESE and EFR was diagnosed as mesenchymal tumor and 4 cases as leiomyoma, among which 34 cases were in fundus ventriculi, 30 in corpus ventriculi and 16 in antrum. Endoscopic closure is significantly lower than laparoscopic closure in operation time span (endoscopic closure VS laparoscopic repair $74.70 \pm 23.55 \mathrm{~min}$ vs $178.35 \pm 38.98 \mathrm{~min}$, $p<0.001$ ) , medical expenses (endoscopic closure VS laparoscopic repair $28463.55 \pm 8228.96 \mathrm{rmb}$ vs $61848.75 \pm 8812.12 \mathrm{rmb}, \mathrm{p}<0.001$ ) and inpatient days (endoscopic closure VS laparoscopic repair $10.50 \pm 3.49$ days vs. $16.95 \pm 4.58$ days, $P<0.01$ ), while there is no significant difference in terms of technical difficulty and effects $(P>0.05)$.

Conclusion: With the training and technical progress of endoscopic closure, it is safe, effective and quick in recovery to have non-laparoscopic assisted surgery on gastric wall closure.

\section{Introduction}

Digestive endoscopy technology advances by leaps and bounds, endoscopic treatment technology from the ESD of endoscopic dissection of the mucosal layer, gradually into the submucosal tumor originated from the intrinsic gastric musculus. From the ntrinsic Muscle Layer Tumor of the gastric mucosa, because the exterior wall type and the mixed wall type were mainly grown outside the cavity of the tumors'site is deep, endoscopic treatment is high risk, difficult and tend to arise intraoperative perforation ${ }^{[1]}$. At the beginning of 2007, some endoscopic doctors tried to remove the lamellar stromal tumor under endoscope and peel off the tumor along the surface of the tumor. The differences in the methods of internal and surgical operations mainly focus on the controversy that to cut off from the edge and from the bottom is positive. According to the principle of the tumor is removed completely, the difficulty of endoscopic resection is that full-layer resection must be perforated. The repair's methods of intraoperative perforation needs to be repaired by laparoscopy in the early stage of endoscopic resection. In 2008, Zhou Pinghong[1, 2] etc. firstly applied ESD technique to treat muscularis propria GIST, and named it endoscopic submucosal excavation(ESE). In 2009, Zhou Pinghong[3] etc. firstly reported endoscopic full thickness resection, (EFR). Once perforation occurs, endoscopic closure is performed quickly with titanium clips and nylon rope. But at this time, perforation is different from complication 
perforation.It is a process and step in endoscopic treatment, which is called therapeutic perforation [46].The endoscopic resection of gastric muscularis propria includes three steps: ESE/EFR, therapeutic perforation and endoscopic perforation closure. Through the endoscopic perforation closure was completed [7-9], the closed wall was missing and the whole operation was completed.By comparing the early laparoscopic repair group with the current endoscopic closure group, in this study we can compare the safety and efficacy of complete resection and closure of gastric musculotoma. Endoscopic doctors pay attention to the training of endoscopic closure, they constantly improve varies endoscopic closures. They expand the scope of endoscopic digestive tract wall treatment, meet the two elements of minimally invasive and safety, and close the mucosal layer to prevent gastric juice from leaking into the abdominal cavity. They observe mucosal closure and promote musculocutaneous healing. These measures are significant.

\section{Materials And Methods}

\subsection{Subjects}

In this study, 40 cases of laparoscopic repair and endoscopic closure treatments, were included in this study. The differences of operation time and medical expenses between the two closure methods were compared and analyzed. This study was conducted in accordance with the Declaration of Helsinki and approved by the ethics committee of our hospital. All participants had signed the informed consent.

\subsection{Instruments}

Therapeutic endoscopy includes endoscopy (GIF-H260, Olympus), double-cavity therapeutic gastroscope and double-curved gastroscope. nylon rope, metal titanium clip and titanium clip release device. Endoscope includes water injection endoscope (GIF-H260, Olympus), double-lumen gastroscope and OLYMPUS GIF-XQ 260 electronic gastroscope; nylon rope includes Olympus integrated nylon rope and Leo hook nylon rope; titanium clip includes titanium clip release device (HX-1 10ur; Olympus)), disposable closed titanium clip (HX-600-135; Olympus), Nanjing minimally invasive opening and closing harmony clip. ESD related instruments and accessories: argon (ICC-200; ERBE), DUAL knife, transparent cap (Dmur201-11802); Olympus), thermal biopsy forceps (FD-410LR; Olympus), injection needle (NM-4L-1; Olympus), $\mathrm{CO} 2$ injection pump (UCR; Olympus)). Laparoscopic surgical instruments, needle holder for endoscope, 3.0 absorbable suture, etc.

\subsection{The surgery methods}

\subsubsection{Preoperative preparation}

Fasting 8 hours before operation, prophylactic treatment with proton pump inhibitor (PPI) and prophylactic use of antibiotics began 2 hours before operation. To place a gastric tube for continuous negative pressure suction to reduce the flow of gastric acid and contents into the abdominal cavity. To 
clean the umbilical hole to prevent incision infection at the umbilical hole after operation. Intraoperative therapeutic perforation is performed by laparoscopy or endoscopic closure

\subsubsection{Submucosal tumor resection}

The operating room is arranged in a ten thousand level clean double endoscope combined operation room, room temperature $22{ }^{\circ} \mathrm{C} \sim 25^{\circ} \mathrm{C}$, humidity $40 \% \sim 60 \%$, endoscopy combined with laparoscopic surgery under general anesthesia intubation, patients take the left side of supine head. During operation we should monitor vital signs and $\mathrm{CO}_{2}$ partial pressure, and before operation gastric cavity is fully rinsed and gastric fluid is absorbed. The pneumoperitoneum pressure was monitored during therapeutic perforation. Once we found the abdominal wall bulge and drum sound, we should used $20 \mathrm{ml}$ injection needle to reduce the abdominal pressure immediately. The puncture point is located below the right costal margin and placed through the abdominal cavity until the perforation is completely closed. it is confirmed that no air is discharged from the exhaust needle and the exhaust needle is pulled out when the pneumoperitoneum is obviously improved. Then, medical treatment to reduce the risk of postoperative peritonitis. ESE tumor exhumation and EFR gastric wall tumor resection. ESE tumor exhumation takes a long time and is carefully peeled off along the surface of the tumor. The perforation is often small and easy to be closed directly by titanium clip.The perforation of the EFR gastric wall tumor resection is large and obvious. And pneumoperitoneum appears rapidly, so it is necessary to monitor the abdominal exhaust of perforation.

\subsubsection{Laparoscopic repair}

It is difficult to repair the perforation closure, and the endoscopic closure level is low in early endoscopic surgery. Therefore, the safe and reliable surgical plan is to choose the double-mirror combined operation laparoscopic-assisted repair. Laparoscopic repair of gastric perforation: compared with traditional surgery, laparoscopic repair of gastric perforation is less trauma, less pain, quick recovery and so on. The time-consuming operation includes laparoscopic search for perforation and repair of perforation. Surgical members includes doctor, assistants and instrument nurses, and the cost includes laparoscopic equipment, repair of perforation, abdominal drainage tube and so on. Operating method, a $1 \mathrm{~cm}$ arc incision was made at the inferior edge of the umbilical cord to reach the subcutaneous. when the pneumoperitoneum was enough, the pneumoperitoneum needle was pulled out, the cannula needle was punctured for $10 \mathrm{~mm}$ and placed into the laparoscopic. Under laparoscopic direct vision, $10 \mathrm{~mm}, 5 \mathrm{~mm}$ and $5 \mathrm{~mm}$ trocars were punctured at $3 \mathrm{~cm}$ below xiphoid process, midline of clavicle, axillary front and costal margin. Insert the appropriate equipment. Firstly, the gastric contents and exudate were absorbed with an attractor, and after the perforation was found, the full-thickness suture was done with 3.0 absorbable suture, now needn't ligation ,and the free part of the greater omentum was stuffed into the perforation, and then knotted. Then, rinse abdominal cavity and pelvic cavity. A drainage tube is placed near the perforation, which is drawn out of the body from the anterior axillary line sleeve and fixed. Finally, to release $\mathrm{CO} 2$ gas, pull out the casing and glue the skin incision.

\subsection{Statistical analysis}


Based on R (edition3.5.1,www.r-project.org), we made the collection and analysis of this experiment. According to the double-sample t-test, the differences of operation time span, medical expenses and inpatient days between the two closure methods were compared. And used the chi-square test to compare the difference in terms of operational difficulty and effects. When $\mathrm{P}<0.05$, we think this difference is significant.

\section{Results}

\subsection{The general characteristics}

The cases of the during endoscopic resection is submucosal tumours (SMT) of the stomach, which were found by the Digestive Endoscopy Center of Affiliated Hospital of Jiangnan University under endoscopic ultrasonography (EUS) during Jan. 2014 to Dec. 2019. The cases'perforation rate is $17 \%(80 / 450)$. Each group included 40 cases aged $30-81$ years, including 48 males and 43 females. Their average age is (55.3+-4.5).

\subsection{The surgery efficacy}

Under general anesthesia with endotracheal intubation we finished 36 patients with ESE and 44 patients with EFR. On the second day after surgery, the patients can active beside the bed. Two days later, their drainage tubes and gastric tubes will be removed. 3-5days later, they can discharge.

The time of the ESE/EFR was $45-122 \mathrm{~min}$. The average time of surgery was $86.8 \mathrm{~min}$. All of them were completely resected at one time, with tumor diameter of $0.6-4.2 \mathrm{~cm}$ and average diameter of $1.8 \mathrm{~cm}$. And there are 40 patients of the laparoscopic repair includes ESE 16 patients and EFR 24 patients. There are 40 patients of the endoscopy closure includes ESE 20 patients and EFR 20 patients. Postoperative pathology of 76 therapeutic cases operated through ESE and EFR was diagnosed as mesenchymal tumor and 4 cases as leiomyoma, among which 34 cases were in fundus ventriculi, 30 in corpus ventriculi and 16 in antrum. We need to use $6 \sim 15$ harmony clips. So the average of titanium clips is 11 .

\subsection{Comparison of laparoscopic repair and endoscopic closure}

We can use endoscopy, metal clips and nylon rings to match both sides of the perforated mucosal edges. Not only can it prevent the gas and digestive fluids, but also it helps to close the perforation and avoid the occurrence of digestive tract fistula. Endoscopic closure is significantly lower than laparoscopic closure in operation time span (endoscopic closure VS laparoscopic repair $74.70 \pm 23.55$ min vs $178.35 \pm$ $38.98 \mathrm{~min}, \mathrm{p}<0.001$ ), medical expenses (endoscopic closure VS laparoscopic repair $28463.55 \pm$ $8228.96 \mathrm{rmb}$ vs $61848.75 \pm 8812.12 \mathrm{rmb}, \mathrm{p}<0.001$ ) and inpatient days (endoscopic closure VS laparoscopic repair $10.50 \pm 3.49$ days vs. $16.95 \pm 4.58$ days, $\mathrm{P}<0.01$ ). According to the "Jiangsu Provincial Guidelines for Classification of Surgery", the difficulty of the two closure methods is level 4 , and there is no difference. According to the operation classification and classification catalogue of the Ministry of 
Health in 2018, the operation difficulty of the two closure methods is level 4, and there is no difference. According to the postoperative follow-up of half a year, there was no recurrence in the patients who underwent both closure methods.

\subsection{Postoperative procedures and follow-up}

No significantly difference was found about complications after surgery between these two kinds of method. CT discovered that the subphrenic free gas was still present in the follow-up for 1 month, and the patient had no symptoms of peritonitis. One patient had postoperative abdominal pain. CT indicated free gas under the diaphragm, and emergency laparoscopic exploration revealed no abdominal exudation. And chronic cholecystitis acutely attacked, and because high-grade mucosal epithelial neoplasia to perform local extensive resection. There was no bleeding or peritonitis. During follow-up, patients' discomfort symptoms disappeared. Gastroscopic reexamination showed that the wound was healed by stellate scar, and there was no residual or recurrence of lesions.

\section{Discussion}

1) Double combined mirror is that laparoscopic repair and endoscopic resection. As for muscularis propria, SMT endoscopic resection ESE/EFR needs skilled endoscopic techniques, and there is a risk that the perforation foci cannot be completely repaired[10-12]. Combined laparoscopic and endoscopic resection (CLER) provides a safety guarantee for patients with endospheric GIsT. When intraoperative acute perforation can't complete closure with endoscopic, we can relay on the laparoscopic repair to assist. Double combined mirror surgery for gastric stromal tumor has the advantages of rapid positioning, optimization of the surgical process, short operative time, small wound, small incision, small risk of exogenous infection, clear operative field, quick recovery, safety and effectiveness. And it is suitable for tumors with a diameter $<5 \mathrm{~cm}$ and difficult to be located by laparoscopy in surgery. However, laparoscopy also has limitations. For example, laparoscopic is difficult to operate when perforation locates in the posterior wall of the stomach. When abdominal pollution is serious and part of the operation needs open, it is higher requirements for the tumor location and the operation group members' cooperation. And it is necessary for experienced doctors to operate.

2) With the development of endoscopic resection, endoscopic perforation closure also appears. With the maturity of endoscopic resection and endoscopic closure, endoscopic muscularis dissection (EMD) includes: endoscopic submucosal excavation (ESE), endoscopic resection of the stomach wall fullthickness EFR, the invisible tunnel submucosal neoplasm STER, etc. They expand the depth and scope of the endoscopic. EFR is the key that the perforation is successfully repaired by microscope in surgery. Titanium clips directly clipped before. As for larger full-thickness defects, firstly, the omentum can be inhaled into the perforated gastric cavity by strong negative pressure suction. And then metal clips clip the omentum and gastric mucosa along the defective edge and effectively suture the defect. We often use titanium clips and nylon rope to suture. The biggest advantage of STER tunnel technology is that not only can it completely remove the intrinsic musculogist at once, but also it can maintain the integrity of the lining mucosa of the digestive tract. Although the perforation occurs in surgery, it can reduce the 
chances of gastrointestinal fistula and intra-abdominal infection by closing the tunnel opening. It is evident that the key of the entire EMD' successful is the site of the intraoperative perforation. At present, with the progress of endoscopic closure, laparoscopic assisted wound sutures gradually reduces and gradually transitions to endoscopic closure [13]. Not only can doctors learn ESD, but also they need to be trained in animal experiments with acute perforation closure and the closure technology is constantly mature. Endoscopic closure reduces laparoscopic staff and instruments, and reduces costs and trauma. The surgery for musculoskeletal tumors will be independently performed by endoscopy. Therefore, double mirror combination is similar to the endoscopic resection alone in trauma, and the postoperative recovery time is no different from endoscopy. Endoscopic closure needs special training. On the one hand, young digestive endoscopy doctors are enthusiastic in learning ESD and other technologies; on the other hand, there is still a lack of special training on ESD and acute perforation disposal ability. Therefore, endoscopic closure of the acute perforation ability needs to be improved rapidly. In the clinical, application of acute digestive tract perforation endoscopic closure is still facing many problems. So before this technology skillfully apply into the clinic, "ESD practice of intraoperative acute perforation endoscopic closure" animal experimental study. "ESD and Perforation repair" training animal experiment design is very practical. Endoscopic doctors learn technology from unfamiliar to familiar, and they need standardized technical operation training. They will be evaluated safety and feasibility. And they accumulate experience and innovate constantly so that they can improve the endoscopic diagnosis and treatment effect. This project is funded by Wuxi Science and Technology Committee, and it provides practical experience and theoretical basis in the study of acute digestive tract perforation for clinical practice.

3) According to the predictability of perforation: it includes complications and therapeutic perforations. With the improvement of operating instruments and endoscopy, a minimally invasive new era must will be brought by endoscopic closure. The passive perforation changes into active perforation, and the perforation becomes a step of endoscopic resection, and we can finish the various operations of the gastrointestinal tract with endoscopic closure. Because gastroenteroscopy is performed on an empty stomach, as long as it is discovered in time and closed quickly with endoscopy. And the symptoms of acute perforative peritonitis are mild, traditional transabdominal surgery can be avoided. What's more, it can reduce the psychological and economic burden of both doctors and patients, shorten the course of disease, reduce the cost of hospitalization, and improve the cure rate quickly. There is no significant difference in the treatment of upper gastrointestinal musculoskeletal tumor with END between endoscopic closure and laparoscopic repair. And It has the advantages of less trauma, faster recovery and lower cost. With the development of endoscopic closure of io-genic gastrointestinal perforation, the indications of endoscopic resection will continue to expand and it has good clinical developed value. It provides a evidence for the clinical application of endoscopic closure of acute gastric perforation. With the development of endoscopic suture technology, many perforations were treated by laparoscopy bofore, now It can be completed by endoscopic suture. And the application of LAET will be gradually reduced and may even be replaced by EFR and STER. However, the training and promotion of endoscopic closure will need the invention and application of a variety of simple endoscopic closure instruments. 


\section{Abbreviations}

SMT: submucosal tumours

ESE: endoscopic submucosal excavation

PPI: proton pump inhibitor

ESD: Endoscopic submucosal dissection

EFR: endoscopic full-thickness resection

EUS: endoscopic ultrasonography

CLER: Combined laparoscopic and endoscopic resection

EMD: endoscopic muscularis dissection

STER: Submucosal Tunneling Endoscopic Resection

\section{Declarations}

\section{Ethics approval and consent to participate:}

This study was conducted in accordance with the Declaration of Helsinki and approved by the ethics committee of our hospital.

\section{Consent to publication:}

All authors final approval of the version to be published.

\section{Availability of data and materials:}

The datesets used or analyzed during the current study are available from the corresponding author on reasonable request.

\section{Competing interests:}

All of the authors had no any personal, financial, commercial, or academic conflicts of interest separately.

\section{Fund projects:}

"Six Talent peak" funded project NO. 2014-WSN-068 in Jiangsu Province, Wuxi Science and Technology Bureau project science and Technology Development fund funded CSE31N1403.

XiCai industry and commerce (2019) 92 Wuxi enterprise academician workstation Project No. C2019301 


\section{Conflict of Interests statement:}

All of the authors had no any personal, financial, commercial, or academic conflicts of interest separately.

\section{Authors Contribution:}

Conception and design of the research: Qiao Q. Acquisition of data: Qiao Q, Tu HM. Analysis and interpretation of the data: Fei BJ, Xu KB. Statistical analysis : Yang F. Obtaining financing : None. Writing of the manuscript : Qiao Q, Li J and Gao QZ. Critical revision of the manuscript for intellectual content : Qiao Q, Tu HM.

\section{Acknowledgements:}

None.

\section{References}

1. Zhou PH, Yao LQ, Qin XY. Endoscopic submucosal dissection for 20 cases of gastrointestinal stromal tumors[J]Chinese Journal of gastrointestinal surgery,2008(11): 219-222.

2. Nobutsugu Abe, Hirohisa Takeuchi, Osamu Yanagida, Tadahiko Masaki, Toshiyuki Mori, Masanori Sugiyama, Yutaka Atomi, Endoscopic full-thickness resection with laparoscopic assistance as hybrid NOTES for gastric submucosal tumor[J] Surg Endosc. 2009 Aug;23(8):1908-13. doi: 10.1007/s00464-008-0317-y. Epub 2009 Jan 30.

3. Zhou PH, Yao LQ, Qin XY, et al. Laparoscopic assisted endoscopic full-thickness resection for gastric submucosal tumors originating from muscularis propria[J]Chinese Journal of digestive endoscopy, 2009, 26(12): 617-621.

4. Xu MD, Yao LQ, Zhou PH, et al. Endoscopic tumor resection via submucosal tunnel for upper gastrointestinal submucosal tumors originating from muscularis propria[J] Chinese Journal of digestive endoscopy, 2011, 28(11): 606-610.

5. Haiqin Wang, Yuyong Tan, Jirong Huo, Deliang Liu囚Submucosal 1-tunnel endoscopic resection for treating upper gastrointestinal multiple submucosal tumor originating from the muscularis propria layer: A report of 12 cases[J]Observational Study,Medicine (Baltimore), 2019,Feb;98(6):e14484. doi: 10.1097/MD.0000000000014484.

6. Jain D, Desai A, Mahmood E, Singhal S.Submucosal tunneling endoscopic resection of upper gastrointestinal tract tumors arising from muscularis propria [J]Ann Gastroenterol. 2017;30(3):262272. doi: 10.20524/aog.2017.0128. Epub 2017 Feb 10.

7. Ling TS, Pei QS, Lv Y, et al. Evaluation of the efficacy and safety of endoscopic resection of giant gastric stromal tumors[J]Chinese Journal of digestive endoscopy, 2013, 30(2): 90-93.

8. Tu HM. Clinical study on minimally invasive treatment of esophageal leiomyoma with gastroscope, endoscopic ultrasonography and thoracoscopy[J]Chinese Medicine, 2011, 6(1): 65-66. 
9. Tu HM. Preliminary application of endoscopy combined with thoracoscope and laparoscopy in the treatment of benign tumors of digestive tract[J] Chinese Journal of digestive endoscopy, 2010, 27(7): 365-366.

10. Isao Nozaki, Junki Mizusawa, Ken Kato, Hiroyasu Igaki, Yoshinori Ito, Hiroyuki Daiko, Masahiko Yano, Harushi Udagawa, Satoru Nakagawa, Masakazu Takagi, Yuko Kitagawa.Impact of laparoscopy on the prevention of pulmonary complications after thoracoscopic esophagectomy using data from JCOG0502: a prospective multicenter study.Multicenter Study[J]Surg Endosc,2018 Feb;32(2):651-659. doi: 10.1007/s00464-017-5716-5. Epub 2017 Aug 4.

11. Tu HM, Qiao Q, Xu Kw, Fei BJ, Yang F, Li J, Gao QZ.Comparative study of endoscopic closure and laparoscopic repair of acute perforation[J]World Cancer Research, 2018, 8(2): 75-80. https://doi.org/10.12677/WJCR.2018.82012

12. Zhou, P.H., Yao, L.Q., Qin, X.Y., et al. (2011) Endoscopic Full-Thickness Resection without Laparoscopic Assistance for Gastric Submucosal Tumors Originated from the Muscularis Propria. Surgical Endoscopy, 25, 2926-2931.

13. Ma LL, Chen SY, Zhou PH, et al. Evaluation of four endoscopic nylon rope ligation in the treatment of upper gastrointestinal submucosal tumors[J] Chinese Journal of digestive endoscopy, 2010, 27(11): 581-584.

\section{Table}

Table 1

The comparison of endoscopic closure and laparoscopic repair with acute perforation

\begin{tabular}{|llll|}
\hline \multicolumn{3}{|c|}{ Operation methods } & P \\
\cline { 2 - 3 } & Laparoscopic repair & Endoscopy closure & \\
\hline Operation time span(min) & $178.35 \pm 39.98$ & $74.7 \pm 23.55$ & $<0.001$ \\
\hline Operation difficulty & Level 4 & Level 4 & - \\
\hline inpatient days (day) & $16.95 \pm 4.58$ & $10.5 \pm 3.449$ & $<0.001$ \\
\hline medical expenses(yuan) & $61848.75 \pm 8812.12$ & $28463.55 \pm 8228.96$ & $<0.001$ \\
\hline
\end{tabular}

\section{Figures}



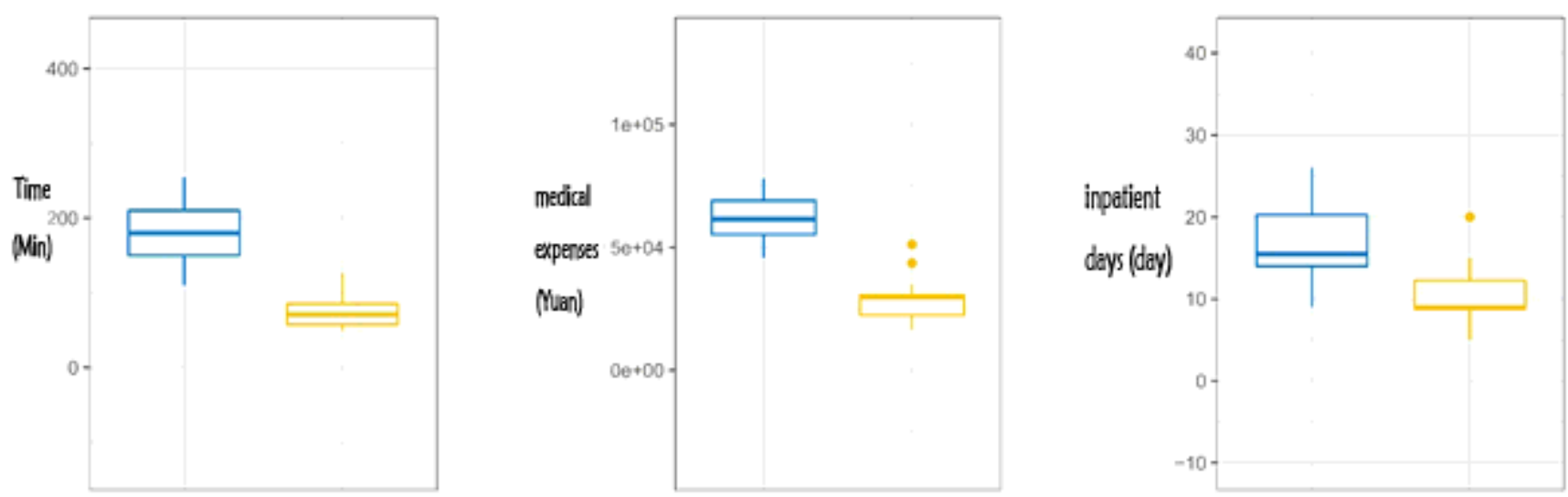

帛 Laparoscopic repair Đ Endoscopy dosure

\section{Figure 1}

Comparison of operation time span, medical expenses and inpatient days between peritoneal repair and endoscopy closure. 